\title{
Expériences de Max Havelaar dans la commercialisation en Suisse de produits en provenance de pays en développement
}

\section{Rolf Buser et Monika Schmutz Cattaneo}

\section{(2) OpenEdition}

\section{Journals}

Édition électronique

URL : http://journals.openedition.org/aspd/1525

DOI : 10.4000/aspd. 1525

ISSN : 1663-9669

Éditeur

Institut de hautes études internationales et du développement

\section{Édition imprimée}

Date de publication : 1 mars 1996

Pagination : 257-264

ISSN : 1660-5934

\section{Référence électronique}

Rolf Buser et Monika Schmutz Cattaneo, «Expériences de Max Havelaar dans la commercialisation en Suisse de produits en provenance de pays en développement », Annuaire suisse de politique de développement [En ligne], 15 | 1996, mis en ligne le 16 mai 2013, consulté le 08 septembre 2020. URL: http://journals.openedition.org/aspd/1525; DOI : https://doi.org/10.4000/aspd.1525 


\title{
Expériences de Max Havelaar dans la commercialisation en Suisse de produits en provenance de pays en développement
}

\author{
Rolf Buser \\ Monika Schmutz Cattaneo
}

Après une longue période de gestation, la fondation Max Havelaar (Suisse) a vu le jour en février 1992 à l'initiative des six grandes oeuvres d'entraide suisses que sont Pain pour le prochain, Caritas, Action de carême, EPER, Helvetas et Swissaid. Elle a été créée sur le modèle de la fondation du même nom active depuis 1988 aux Pays-Bas.

Max Havelaar est le titre d'un roman autobiographique du néerlandais Eduard Douwes Dekker, fonctionnaire aux Indes orientales néerlandaises (I'Indonésie actuelle), paru en 1860 . Cet auteur fut le premier à dénoncer certaines pratiques en vigueur dans les colonies européennes, et notamment l'exploitation de la main-d'oeuvre locale dans les plantations de café. II n'est donc pas étonnant que le titre de son livre soit devenu le label du commerce équitable. Le café fut d'ailleurs le premier produit à obtenir le label de la fondation Max Havelaar, et ce, à peine deux mois après que celle-ci ait été créée.

Le but de la fondation Max Havelaar est de promouvoir un commerce humainement équitable et respectueux de l'environnement et d'améliorer l'information du public sur les problèmes de développement y relatifs. Pour ce faire, elle accorde notamment un label aux produits issus de régions en développement et qui satisfont à certains critères minimaux en matière sociale et écologique. La fondation Max Havelaar n'a pas de but lucratif. Elle ne se consacre pas directement à la commercialisation ou à la transformation de produits mais sert d'intermédiaire entre les organisations de producteurs des pays du Sud et le marché suisse, dans le but d'offrir aux premiers un accès direct au second. 
Contre paiement d'une taxe, la fondation attribue aux importateurs/distributeurs du Nord une licence fixant les conditions d'importation des produits Max Havelaar et d'utilisation du label. La fondation sert aussi d'organisme de certification et de contrôle.

La fondation Max Havelaar est financée surtout par ses organisations fondatrices, des contributions de l'Office fédéral des affaires économiques extérieures (OFAEE) et par les recettes des licences délivrées. Les organisations fondatrices ont versé 141'000 francs en 1992, 321'000 francs en 1993 et 306 '000 francs en 1994. L'OFAEE a versé des contributions de $485^{\prime} 000$ francs en 1992, 321'000 francs en 1993 et 305'000 francs en 1994. Les recettes de licences se sont élevées à 303'000 francs en 1992, 424'000 francs en 1993 et 457'000 francs en 1994 (chiffres des rapports annuels).

Grâce aux trois grands distributeurs que sont la Coop, la Migros et Volg, aux torréfacteurs de café, aux importateurs de la première heure (mouvement des Magasins du monde) et à une prise de conscience progressive des consommateurs du Nord en faveur du commerce équitable, le café "solidaire" a conquis une part de marché de $5 \%$ dès sa première année d'existence. Ce résultat dépassait les plus folles espérances tant des commerçants que des responsables de cette initiative. Indiscutablement, de plus en plus de consommateurs du Nord sont disposés à s'associer à des objectifs de politique de développement et de lutte contre la pauvreté en modifiant leur comportement d'achat, du moins tant que la qualité des produits et la bonne affectation du surplus des recettes sont garanties.

\section{Collaboration à l'échelle européenne}

Le succès considérable de la fondation Max Havelaar en Suisse, aux Pays-Bas et en Belgique a conduit à la création d'organisations de commerce équitable dans d'autres pays européens. Max Havelaar (Suisse) collabore étroitement avec toutes les organisations existantes, soit en Belgique, au Danemark, en Allemagne, en France, en Grande-Bretagne, aux Pays-Bas, en Italie, au Luxembourg, en Autriche et même au Canada, aux Etats-Unis et au Japon. Cette collaboration a été institutionnalisée en 1993 par la signature d'un accord de coopération dans le domaine du café, conclu entre les sept initiateurs européens du label pour un commerce équitable. Au cours de l'année 1994, des arrangements correspondants ont été signés pour d'autres produits. Les organisations signataires de ces accords ont adopté:

- des critères de sélection identiques,

- une commission commune chargée de choisir les producteurs,

- des prix minimums et des conditions commerciales uniformes,

- un registre commun de producteurs,

- des principes fondamentaux identiques en matière de gestion,

- une gestion centralisée des registres des producteurs avec banque de données centrale,

- une division du travail aussi avantageuse que possible. 
Dans le cadre de cette répartition du travail, Max Havelaar (Suisse) est responsable des pays andins (Bolivie, Pérou, Equateur, Colombie et Venezuela), pays où les organismes suisses privés et publics d'aide au développement sont particulièrement actifs. La fondation assiste et contrôle les producteurs de café de ces pays et examine les nouvelles demandes d'adhésion. En outre, Max Havelaar (Suisse) a géré jusqu'à mi-1995 le registre international du miel et contrôlé les organisations d'apiculteurs d'Amérique latine. La gestion du registre du cacao est du ressort de la fondation suisse à Bâle depuis le 1er janvier 1995.

\section{Un label pour le miel, le cacao et le sucre}

Encouragée par le succès des ventes de café "solidaire", Max Havelaar (Suisse) a lancé en automne 1993 son deuxième produit, le miel. Outre Caritas et $\mathrm{OS} 3$, qui offraient déjà un vaste assortiment de miels à des prix équitables, la Coop participa à cette action en commercialisant un miel d'Amérique du Sud. En 1994, les ventes de miel portant le label Max Havelaar totalisèrent 228'000 kilos, soit plus de $5 \%$ du miel importé. Sur la totalité du miel consommé en Suisse (en moyenne 6000 tonnes par année, c'est-à-dire plus d'un kilo par personne et par année) entre 50 et $70 \%$, selon la production nationale, est importé.

Le café et le miel répondant aux critères du commerce équitable sont essentiellement importés d'Amérique latine. Quant au cacao, troisième produit à porter le label Max Havelaar, il provient surtout d'Afrique, plus précisément du Ghana, du Cameroun et de la Sierra Leone, déchirée par la guerre civile. Partenaire de longue date du commerce équitable traditionnel et fournisseur de cacao certifié biologique, la coopérative bolivienne El Ceibo a aussi été intégrée au registre des producteurs de Max Havelaar, de même qu'une coopérative équatorienne. Les 14 chocolats suisses Max Havelaar satisfont également à une exigence longtemps réclamée par les oeuvres d'entraide en matière de politique de développement: ils sont fabriqués avec du sucre brut commercialisé à des conditions équitables et raffiné dans le pays d'origine. Ce chocolat nouvelle formule (cacao solidaire + sucre brut solidaire + lait suisse) a été présenté aux consommateurs en novembre 1994. La réaction fut prometteuse: même le pionnier de l'industrie chocolatière Maestrani rejoignit les preneurs de licence Coop, Migros et OS3. Après une année, la part de marché s'élève à environ $1 \%$, ou 400 tonnes (4 millions de plaques). Trois raisons peuvent expliquer ce moindre succès par rapport aux autres produits. Premièrement, l'assortiment de chocolat est beaucoup plus vaste que celui de café (environ 50 plaques de chocolat par grand distributeur), deuxièmement, le chocolat est un produit de luxe pour lequel les consommateurs font preuve d'une grande fidélité au marché et troisièmement, les grands distributeurs que sont Migros et Coop ont commencé par ne proposer respectivement qu'une et deux plaques Max Havelaar seulement. 
Les conditions exigées par Max Havelaar pour l'importation de café, de miel, de cacao et de sucre sont adaptées aux problèmes et à l'infrastructure des petits producteurs et de leurs coopératives. Voici quelques exemples de ces critères pour ce qui concerne le café.

1. Le café provient de coopératives de petits producteurs soigneusement sélectionnées (on évite les intermédiaires locaux qui agissent souvent en exploiteurs).

2. L'achat d'une quantité fixe de café est garantie et une partie de la récolte est payée d'avance.

3. Le café est cultivé et traité de façon aussi respectueuse de l'environnement que possible.

4. Les producteurs de café obtiennent une rémunération leur permettant de mener une vie décente (le prix plancher Max Havelaar est de 1,26 US\$/livre pour le café traditionnel et de 1,41 US\$/livre pour le café certifié biologique). Si le prix du marché est supérieur au prix garanti par Max Havelaar, l'association de producteurs obtient une prime supplémentaire de 5 cents US pour le café traditionnel et de 15 cents US pour le café biologique.

Lorsqu'une organisation de producteurs satisfait aux critères de la fondation Max Havelaar en matière tant commerciale que de politique de développement (qualité, fiabilité des partenaires), un contrat de collaboration est signé. Celui-ci régit notamment les éléments suivants.

1. L'organisation de producteurs (une coopérative par exemple) s'engage à prendre des mesures visant à éliminer les monocultures et à diversifier la production (dans le but d'éviter la surproduction).

2. L'affectation des recettes est décidée par voie démocratique au sein de l'organisation.

3. L'organisation doit disposer d'une administration efficace et transparente ainsi que d'un organe de contrôle.

4. L'organisation doit être disposée à accepter de nouveaux membres.

5. L'organisation favorise tout ce qui peut renforcer son autonomie ainsi que le processus de développement régional (stockage, finition, emballage, transport, crédit, formation et prévoyance en matière de santé).

6. L'organisation propose à ses membres des crédits à des taux d'intérêt favorables.

\section{Elargissement du concept}

La certification du quatrième produit, le thé, a nécessité des travaux de préparation intensifs. Bien plus que le simple lancement sur le marché d'un produit exotique classique à des conditions équitables, elle a requis un élargissement du concept Max Havelaar. En effet, le thé provenant de petits producteurs n'est disponible qu'en très petite quantité dans une qualité satisfaisant aux exigences 
du marché européen. Ce phénomène est dû, d'une part au fait que les gigantesques plantations de thé créées par les colons britanniques en Inde, au Sri Lanka et en Afrique orientale ont été reprises par les autochtones après l'indépendance, et d'autre part au fait que le thé doit être traité immédiatement après avoir été cueilli. L'infrastructure nécessaire pour traiter le thé - afin d'assurer la qualité exigée pour l'exportation - ainsi que le conditionnement de celui-ci nécessitent des investissements importants, trop coûteux pour les petits producteurs et donc réservés aux seules plantations ayant un volume de production élevé.

L'élargissement du concept Max Havelaar aux produits provenant de grandes plantations n'a pas affecté les principes fondamentaux du commerce équitable, le but étant toujours de faire bénéficier ceux qui exécutent le travail, à savoir, en l'occurrence, les ouvriers des plantations. Les revenus supplémentaires (2.50 DM - marks allemands - par kilo de thé et $3 \mathrm{DM}$ pour le thé de culture biologique) payés à l'achat du thé Max Havelaar sont versés dans un fonds exclusivement utilisé pour l'amélioration des conditions de travail et de vie des ouvriers des plantations et de leur famille. Un comité réunissant des représentants des employeurs et des employés décide de l'utilisation de cet argent. Les personnes concernées sont en effet les mieux à même d'affecter celui-ci à l'achat de vaches laitières ou de jeux pour des crèches, à l'installation de l'électricité dans les logements, à la construction de maisons communautaires, à l'enseignement d'autres professions aux enfants des travailleurs ou à des projets de protection de l'environnement.

Pour être reconnue par Max Havelaar, une plantation de thé doit satisfaire à de nombreuses exigences. Elle doit notamment s'engager à respecter toutes les dispositions légales et tarifaires minimales en vigueur (selon le BIT) et à garantir une transparence absolue dans tous les domaines. Le respect de ces dispositions est contrôlé sur place à intervalles réguliers. Comme pour les autres produits, la fondation Max Havelaar (Suisse) gère, en collaboration avec les autres organisations de commerce équitable européennes, un registre des producteurs de thé. On y trouve à ce jour des producteurs d'Inde, du Sri Lanka, du Népal et du Zimbabwe (dont un tiers des plantations produit du thé certifié biologique). Par le passé, les preneurs de licence de thé Havelaar en Suisse, à savoir la Coop, la Migros, OS3, Morga, Milliquet et d'autres petits distributeurs, importaient leur thé d'Inde et du Sri Lanka.

Après l'Amérique latine (café, cacao, miel et sucre) et l'Afrique (café et cacao), l'Asie a ainsi renforcé sa présence dans le concept global Havelaar (auparavant, seul le sucre importé des Philippines provenait d'Asie).

\section{5 organisations réparties sur trois continents}

Plus de 100 '000 familles de petits producteurs du monde entier bénéficient de la commercialisation équitable de leurs produits en Europe, le volume d'exportation correspondant étant nettement supérieur à 30 millions US\$. A elle seule, la Suisse a versé plus de 8 millions de francs de recettes supplémentaires aux 
producteurs des pays du Sud. Plus précisément: à 70 organisations de producteurs de café en Bolivie, au Brésil, au Cameroun, en Colombie, au Costa Rica, au Guatemala, en Haïti, au Honduras, au Mexique, au Nicaragua, en Ouganda, au Pérou, en République dominicaine, au Salvador, en Sierra Leone, en Tanzanie, au Venezuela et au Zaïre; à 9 coopératives de producteurs de miel au Chili, au Guatemala, au Mexique et en Uruguay; à 5 associations de producteurs de cacao en Bolivie, en Equateur, au Ghana, au Cameroun et en Sierra Leone; à 4 producteurs de sucre au Costa Rica et aux Philippines, et à 7 plantations de thé en Inde et au Sri Lanka.

\section{Résultats qualitatifs}

Le succès des produits Max Havelaar ne se mesure pas seulement en quantité mais aussi en qualité. Outre l'amélioration du revenu des producteurs, cette initiative engendre en effet les progrès qualitatifs suivants.

1. Le concept du commerce équitable a subsisté chez les producteurs comme sur le marché pendant la période de flambée des prix du café à l'échelle internationale.

Les producteurs ont pris conscience que la stabilité offerte par le concept de commerce équitable était plus importante que la plus-value réalisée et ce, même en temps d'explosion des prix. En effet, lorsqu'une coopérative est en panne de liquidités et ne peut acheter le café de ses membres, la clause de préfinancement de Max Havelaar permet d'éviter un effondrement des prix qui pourrait avoir des répercussions sur une région entière. $A$ cet égard, le réseau des producteurs liés à Max Havelaar joue un rôle d'équilibrage des prix au niveau local et régional. Rien d'étonnant dès lors à ce que les demandes d'adhésion au registre des producteurs ne cessent d'affluer auprès de la fondation. Du point de vue du marché, les ventes de café Max Havelaar n'ont même pas souffert de la hausse des prix du marché mondial et ce, alors que $70 \%$ des consommateurs (d'après les résultats d'une enquête) étaient au courant de ce renchérissement.

2. Max Havelaar a pour objectif d'ouvrir le marché aux producteurs. Les contacts directs entre les organisations de producteurs et les importateurs européens ne débouchent pas seulement sur des contrats conclus aux conditions Max Havelaar. Ils permettent aussi de signer des arrangements aux conditions normales du marché, en évitant les intermédiaires locaux peu scrupuleux.

3. Grâce à la garantie de prix minimum, les importateurs et les torréfacteurs sont en mesure d'exiger une qualité de premier ordre. Cette incitation à favoriser la qualité (plutôt que la quantité) a des conséquences tant microéconomiques (offre de meilleures perspectives d'écoulement) que macroéconomiques (prévient une inondation du marché imputable à l'aggravation de la surproduction). 
4. La prime pour culture biologique et l'incitation à diversifier la production poussent les producteurs à mieux tenir compte de l'environnement. Les partenaires de Max Havelaar sont ainsi toujours plus nombreux à opter pour la culture biologique. En règle générale, les fermes des petits producteurs (cultures mixtes) sont considérées comme étant beaucoup plus respectueuses de l'environnement que les monocultures.

5. Un autre élément a des effets qui sont impossibles à quantifier directement mais qui peuvent se révéler importants à long terme: certains employés des entreprises du Nord (importateurs ou preneurs de licences), se rendant auprès des producteurs du Sud, y découvrent une réalité qui leur laisse de fortes impressions personnelles et qu'ils répercutent ensuite sur leurs entreprises.

6. Le succès du concept Max Havelaar a prouvé que le commerce équitable n'est pas une utopie et a permis de le faire connaître, grâce notarnment à l'écho qu'en a fait la presse. Par ailleurs, cette initiative a déclenché dans d'autres branches un dynamisme visant à lancer un label pour d'autres produits (les fleurs, les textiles (Double Income Project - DIP), les tapis (STEP), par exemple).

\section{Risque d'un foisonnement de labels}

Craignant une surabondance de labels pouvant entraîner une dévalorisation de la notion du commerce équitable, les organisations d'entraide fondatrices de Max Havelaar (Suisse) ont tenté de s'en prémunir: leurs "directives stratégiques" stipulent que Max Havelaar doit rester un des principaux organismes chargés d'attribuer le label de commerce équitable aux produits alimentaires et aux autres produits et que les organismes d'entraide mettront tout en oeuvre pour éviter un foisonnement de labels en Suisse.

Bien entendu, la notion de "commerce équitable" ne peut guère être protégée par une loi ou faire l'objet d'un monopole. Du point de vue de la politique de développement, il serait néanmoins judicieux et souhaitable que cette notion soit définie par les organisations de développement afin d'éviter toute confusion et tout abus. Le conseil de fondation Max Havelaar a d'ores et déjà réagi sur ce point en publiant un document intitulé «Exigences posées au commerce équitable du point de vue de la fondation Max Havelaar". Ce catalogue d'exigences laisse cependant suffisamment de marge de manoeuvre pour des adaptations spécifiques à certains produits.

Le coeur du problème demeure la transparence. Or celle-ci peut très bien être garantie par un label (clairement différencié du label de vente, comme c'est le cas pour la récente initiative STEP) attribué par une instance de certification indépendante.

$\mathrm{Si}$ les divergences entre concept et contenu sont expliquées par le biais d'une politique de communication et de relations publiques adéquate, alors le risque de confusion (pouvant entraîner un certain scepticisme à l'égard du commerce équitable) est considérablement amoindri, à défaut d'être éliminé. 


\section{Perspectives}

La fondation Max Havelaar s'efforcera d'étendre son droit de pionnier et de principal organe d'attribution de label tout en maintenant un niveau d'exigence élevé pour tous les nouveaux produits Havelaar.

L'accent sera mis sur les produits présentant un chiffre d'affaires potentiel élevé, offrant des atouts en matière de politique du développement et pouvant faire l'objet d'un message facilement transmissible aux consommateurs. En outre, le rapport entre les dépenses (développement des produits, surveillance des producteurs, prospection du marché) et les revenus (pour les producteurs et pour la fondation) doit être sain.

En ce qui concerne le choix de nouveaux produits, la répartition des coûts et du travail dans le cadre de la coopération européenne pour l'attribution du label joue également un rôle prépondérant. A l'instar des produits Max Havelaar actuels (café, miel, sucre, cacao et thé) les nouveaux produits devront être "eurocompatibles" (harmonisation des critères applicables au commerce équitable et des critères de sélection des producteurs).

Dans la perspective du marché unique européen, il est important d'éviter une diversité d'exigences nationales (comme pour les écolabels) qui peut produire une distorsion de la concurrence. L'instauration d'un label européen unique constitue d'ores et déjà un objectif à moyen terme.

\section{Conclusion}

Le commerce équitable, à savoir le commerce de produits respectant l'homme et l'environnement, a le vent en poupe. La politique d'un commerce Nord-Sud fondé sur des coûts réels commence à prendre forme. A cet égard, Max Havelaar a posé quelques jalons mais il reste encore beaucoup à faire, notamment en matière de prospection, pour ancrer et développer cette pratique sur un marché où règne une concurrence acharnée. 